\title{
Oral lichen planus (OLP), oral lichenoid lesions (OLL), oral dysplasia, and oral cancer: Retrospective analysis of clinicopathological data from 2002-2011
}

Casparis, S ; Borm, J M ; Tektas, S ; Kamarachev, J ; Locher, M C ; Damerau, G ; Grätz, K W ; Stadlinger, B

\begin{abstract}
INTRODUCTION: This 10-year retrospective study analyzed the incidence of malignant transformation of oral lichen planus (OLP). The study also included dysplasia and oral lichenoid lesion (OLL) in the initial biopsy as a potential differential diagnosis. MATERIAL AND METHODS: A total of 692 scalpel biopsies were taken from 542 patients (207 [38.2\%] men and 335 [61.8\%] women). Clinical and histopathological parameters were analyzed. RESULTS: The parameters gender $(\mathrm{p}=0.022)$ and smoking behavior $(\mathrm{p}<0.001)$ were significantly associated with the severity of diagnosis. Mucosal lesions with an ulcerative appearance $(\mathrm{p}=0.006)$ and those located on the floor of the mouth $(\mathrm{p}<0.001)$ showed significantly higher degrees of dysplasia or were diagnosed as oral squamous cell carcinoma (OSCC). Smoking and joint disease appeared to be significant risk factors. Treatment with tretinoin in different concentrations (0.005-0.02 \%) significantly improved diagnosis. Twelve patients ( 8 female, 4 male) showed malignant transformation to OSCC within an average period of 1.58 years. The malignant transformation rate (MTR) was higher for OLL $(4.4 \%)$ than OLP $(1.2 \%)$. If the first biopsy showed intraepithelial neoplasia, the risk of developing OSCC increased (by $3.5 \%$ for squamous intraepithelial neoplasia (SIN) II and by $6.7 \%$ for SIN III). CONCLUSION: Although we cannot rule out that OLP is a premalignant oral condition, we can confirm that OLP had the lowest MTR of all diagnoses.
\end{abstract}

DOI: https://doi.org/10.1007/s10006-014-0469-y

Posted at the Zurich Open Repository and Archive, University of Zurich

ZORA URL: https://doi.org/10.5167/uzh-102729

Journal Article

Published Version

Originally published at:

Casparis, S; Borm, J M; Tektas, S; Kamarachev, J; Locher, M C; Damerau, G; Grätz, K W; Stadlinger, B (2015). Oral lichen planus (OLP), oral lichenoid lesions (OLL), oral dysplasia, and oral cancer: Retrospective analysis of clinicopathological data from 2002-2011. Oral and Maxillofacial Surgery, 19(2):149-156. DOI: https://doi.org/10.1007/s10006-014-0469-y 


\title{
Oral lichen planus (OLP), oral lichenoid lesions (OLL), oral dysplasia, and oral cancer: retrospective analysis of clinicopathological data from 2002-2011
}

\author{
S. Casparis • J. M. Borm • S. Tektas • J. Kamarachev • \\ M. C. Locher • G. Damerau • K. W. Grätz • B. Stadlinger
}

Received: 24 October 2013 / Accepted: 6 October 2014 / Published online: 14 October 2014

(C) Springer-Verlag Berlin Heidelberg 2014

\begin{abstract}
Introduction This 10-year retrospective study analyzed the incidence of malignant transformation of oral lichen planus (OLP). The study also included dysplasia and oral lichenoid lesion (OLL) in the initial biopsy as a potential differential diagnosis.

Material and methods A total of 692 scalpel biopsies were taken from 542 patients (207 [38.2 \%] men and 335 [61.8\%] women). Clinical and histopathological parameters were analyzed.

Results The parameters gender $(p=0.022)$ and smoking behavior $(p<0.001)$ were significantly associated with the severity of diagnosis. Mucosal lesions with an ulcerative appearance $(p=0.006)$ and those located on the floor of the mouth $(p<0.001)$ showed significantly higher degrees of
\end{abstract}

S. Casparis $(\triangle) \cdot$ J. M. Borm $\cdot$ S. Tektas $\cdot$ M. C. Locher $\cdot$

G. Damerau $\cdot$ K. W. Grätz $\cdot$ B. Stadlinger

Department of Cranio-Maxillofacial and Oral Surgery, University

Hospital Zurich, Plattenstrasse 15, 8032 Zurich, Switzerland

e-mail: stephanie.casparis@hotmail.com

J. M. Borm

e-mail: jan.borm@zzm.uzh.ch

S. Tektas

e-mail: sibel.tektas@hotmail.com

M. C. Locher

e-mail: michael.locher@zzm.uzh.ch

G. Damerau

e-mail: georg.damerau@zzm.uzh.ch

K. W. Grätz

e-mail: klaus.graetz@usz.ch

B. Stadlinger

e-mail: bernd.stadlinger@zzm.uzh.ch

J. Kamarachev

Department of Dermatology, University Hospital of Zurich,

Rämistrasse 100, Zurich 8091, Switzerland

e-mail: jivko.kamarachev@usz.ch dysplasia or were diagnosed as oral squamous cell carcinoma (OSCC). Smoking and joint disease appeared to be significant risk factors. Treatment with tretinoin in different concentrations (0.005-0.02\%) significantly improved diagnosis. Twelve patients ( 8 female, 4 male) showed malignant transformation to OSCC within an average period of 1.58 years. The malignant transformation rate (MTR) was higher for OLL $(4.4 \%)$ than OLP $(1.2 \%)$. If the first biopsy showed intraepithelial neoplasia, the risk of developing OSCC increased (by $3.5 \%$ for squamous intraepithelial neoplasia (SIN) II and by $6.7 \%$ for SIN III).

Conclusion Although we cannot rule out that OLP is a premalignant oral condition, we can confirm that OLP had the lowest MTR of all diagnoses.

Keywords Oral lichen planus · Dysplasia $\cdot$ Malignant transformation $\cdot$ Oral squamous cell carcinoma

\section{Introduction}

Oral lichen planus (OLP) is a chronic inflammatory disease. Commonly, OLP affects the oral mucosa, but it can involve also other tissues like the skin, genital mucosa, and nails. Oral lesions usually appear at the buccal mucosa, tongue, gingiva, palate, and lips [1]. The reported prevalence rates vary from $0.1-4 \%$ of the population; it affects middle-aged people and is more common among women [2]. OLP is divided into six different forms: the best known, reticular type; the papular type; the "plaque-like" type; the atrophic type; the ulcerative type; and the rare bullous type [3]. The atrophic, ulcerative, and bullous types commonly are grouped together under the term "erosive type." The reticular, popular, and plaque-like types often go unnoticed by patients because they are normally asymptomatic, whereas the erosive type is accompanied by 
complaints such as burning, pain — especially when eatingand changes in taste.

Although the pathogenesis of OLP is not fully understood, evidence indicates that an imbalance of immunologic cellular reactivity is central; however, the pathogenesis of OLP is considered also to be multifactorial. Histologically, classic features are typical for OLP: hyperkeratosis, liquefactive degeneration of the basal epithelial cells, colloid bodies, and the presence of a well-defined band-like zone of cellular infiltration [4]. This inflammatory infiltrate is confined to the superficial part of the connective tissue and consists mainly of lymphocytes. Studies have verified that the majority of $\mathrm{T}$ lymphocytes found in the epithelium or near damaged basal keratinocytes are activated CD8 $+\mathrm{T}$ lymphocytes, whereas CD4+ cells often occur in small clusters deeper down in the subepithelial lymphocyte-rich band [5]. CD8+ T lymphocytes are cytotoxic cells that trigger the apoptosis of basal keratinocytes. This process is promoted indirectly through an activated CD4+ $\mathrm{T}$ cell response [6] or directly through antigen-presenting keratinocytes $[7,8]$ or through a combination of both. However, the nature of the antigen is uncertain. Furthermore, many factors are known to influence OLP in a causative or aggravating way; these factors include the following (see also the review by Ismail et al. [9]): different drugs, dental materials, chronic liver disease and hepatitis $\mathrm{C}$ virus, psychological factors, genetics, tobacco chewing, and graft-versus-host disease.

Another issue discussed in relation to OLP is its potential towards malignancy. The possible malignant transformation is discussed in several prospective $[10,11]$ and retrospective studies [12-14]. These studies reported transformation rates varying from $0-9 \%$. As demonstrated by Bornstein et al. [15], it is absolutely necessary to verify the initial diagnosis with a biopsy and compare developed oral squamous cell carcinoma (OSCC) to the original anatomical region of the OLP. Moreover, it must be noted whether dysplasia was present at the initial diagnosis.

This retrospective study presents patients seen at the Clinic of Oral Surgery, University of Zurich, who were clinically and histopathologically diagnosed with OLP or oral lichenoid lesions (OLL) in the years 2002 to 2011. The clinical and histological data from the initial diagnosis were compared to the changes during follow-up.

\section{Material and methods}

This retrospective study was performed on a sample of 542 patients diagnosed with OLP or OLL during the period 2002 to 2011. The diagnosis of OLP was based on the identification of the clinical lesions and the histopathological findings. A total of 692 biopsies were taken. All patients were diagnosed and treated at the Clinic of Oral Surgery, University of Zurich.
A complete clinical work-up was performed on the basis of the patients' history (gender, age at the time of biopsy, liver disease, joint disease, immune suppression, mental disorder, history of tumor, diabetes, medications, alcohol consumption, smoking), clinical aspects of the lesion (appearance and sites of oral involvement), and histological diagnosis. The severity of illness increased from OLP or OLL to dysplasia to OSCC. For dysplasia, the squamous intraepithelial neoplasia (SIN) classification (I-III) was used. Furthermore, we noted the development of the OLP and OLL during the follow-up period and treatment. According to the symptoms of the mucosal lesion, patients were treated with corticosteroids (topical or systemic) or retinoids (topical) or both. Patients who did not fulfill the clinical and histological criteria for OLP and OLL were excluded from the analysis. When calculating the malignant transformation rate (MTR), we excluded six cases that initially contained OSCC as a diagnosis. The remaining cases of OSCC were compared to their initial diagnoses. This was calculated within the follow-up group as well as within the total number of biopsies. All patients had regular follow-up examinations after the first biopsy and were clinically inspected at the Clinic of Oral Surgery. The first biopsy was always taken in order to enable a primary histopathological diagnosis. Biopsies during follow-up examinations were only taken when there was a clinical suspicion.

Every OSCC that was diagnosed in this period of time (2002-2011) was double checked retrospectively regarding a former diagnosis of OLP, OLL or dysplasia. Additionally the follow-up biopsies were taken from the clinically suspicious regions and do not always match the location of the first biopsy.

The statistical analysis of the data was performed with the Statistical Package for Social Sciences (SPSS), version 18. A descriptive study was made of each variable. The MannWhitney $U$ test and the Kruskal-Wallis test were used for non-parametric variables. Results were considered significant when $p<0.05$. Parameters affecting the longitudinal course of illness severity were calculated by mixed models.

\section{Results}

Data from a total of 542 patients (207 [38.2\%] men and 335 [61.8\%] women) were analyzed retrospectively. The median age was 58.0 years (SD 13.95); the youngest patient was 15 years old, and the oldest was 98 years old. Table 1 shows the sociodemographic characteristics and the most prevalent systemic disorders found in the patients.

Statistical analysis found several factors that correlated significantly with the diagnosis of the mucosal biopsies in our patients. Gender had a significant effect on the diagnosis: men had a greater severity of illness $(p=0.022)$ than women, although samples were taken more often from women. 
Table 1 Patient characteristics

\begin{tabular}{llll}
\hline Variables & Categories & Frequency $(n)$ & Percentage (\%) \\
\hline Gender & Male & 207 & 38.2 \\
& Female & 335 & 61.8 \\
Median Age 58 years & Range 15-98 & & \\
Positive history for: & & & 12.4 \\
Joint disease & & 67 & 4.2 \\
Liver disease & & 23 & 6.3 \\
Diabetes & & 34 & 1.7 \\
Immune suppression & 9 & 5.0 \\
History of tumor & 27 & 6.1 \\
Mental disorders & 33 & 26.0 \\
Smoking & & 141 & 10.5 \\
Alcohol consumption & 57 & 26.0 \\
Allergy & & 146 & $<0.001$ \\
\hline
\end{tabular}

Statistical analysis was performed with the Mann-Whitney $U$ test. Results were considered significant when $p<0.05$

Furthermore, smokers were more likely to have a dysplastic lesion $(p<0.001)$, whereas alcohol consumption showed only a trend towards significance $(p=0.054)$.

When comparing the different diagnoses with the clinical parameters of the mucosal lesion, the appearance and localization of the mucosa were found to be significant. The ulcerative form resulted in greater severity $(p=0.006)$ of illness, and the floor of the mouth was affected significantly more often $(p<0.001)$ by more severe diagnosis than other oral regions.

A total of 692 biopsies were taken from the patients. To describe the results of the biopsies, we separated the first biopsy from the follow-up biopsies: 565 (82\%) samples were taken as a first biopsy and $127(18 \%)$ were taken in the follow-up. Eighty-seven patients were biopsied twice; 22, three times; 12, four times; and 6, 5 times. In total, we took almost two thirds of all biopsies (60.3\%) from women and just over one third (39.7\%) from men. The gender distribution changed at follow-up; almost as many follow-up biopsies were taken from men $(44.88 \%)$ as from women $(55.12 \%)$. This development is due to the fact that men had the more severe diagnoses (Fig. 1) and therefore needed several successive biopsies.

Figure 2 gives an overview of the clinical appearance of the mucosal lesions described by different physicians. All types were found among both the first and follow-up biopsies, but the proportion of benign, reticular, and lichenoid variants was lower at follow-up, whereas the proportion of ulcerative lesions was higher.

The first biopsies were taken predominantly from the buccal mucosa $(54.46 \%)$, ridge $(22.87 \%)$, and border of the tongue $(16.09 \%)$, whereas the follow-up biopsies were taken more often from the floor of the mouth $(14.75 \%)$ and border of the tongue $(29.51 \%)$. The palate was affected in only $2-$ $3 \%$ of the cases (Fig. 3).

Figure 4 shows the diagnoses of the biopsy results. The benign lesions, including OLP and OLL, accounted together for $85.48 \%$. Dysplasia was found in $12.93 \%$, carcinoma in situ in $0.53 \%$, and OSCC in $1.06 \%$ of the first biopsies. At follow-up, we found a large increase in the proportion of dysplasia (48.03\%), particularly SIN I and SIN II. The OSCC also increased to $8.66 \%$ at follow-up.

Age and gender had no significant effect on the progression of OLP and OLL towards dysplasia and OSCC, even though men had more serious diagnoses than women. However, the exacerbation was significantly associated with smoking $(p<0.001)$ and, interestingly, with the presence of joint disease $(p=0.039)$.

Fifty percent of the patients were treated with corticosteroids, mostly topical; $38(6.9 \%)$ of the first biopsy group and $11(10.8 \%)$ of the follow-up group were treated also with retinoids. Patients who received retinoids showed a significant improvement in diagnosis $(p=0.017)$ towards a milder degree of dysplasia. Surgical treatment was necessary in $7.75 \%$ of cases in total. One third of the follow-up biopsied regions needed to be removed surgically.

In the present study, the average MTR of the mucosal lesions into OSCC was $2.15 \%$ : five cases of OLL and five cases of OLP developed directly OSCC. Only one case of SIN II and one case of SIN III developed cancer. Table 2 presents the average MTR in more detail and shows that OLL had a higher MTR than OLP. If intraepithelial neoplasia was present in the first biopsy, the risk of developing OSCC strongly increased for SIN II and SIN III (by $9.1 \%$ ); there was no transformation of a SIN I case. 
Fig. 1 Gender distribution in relation to the shift between the first biopsies and the follow-up biopsies

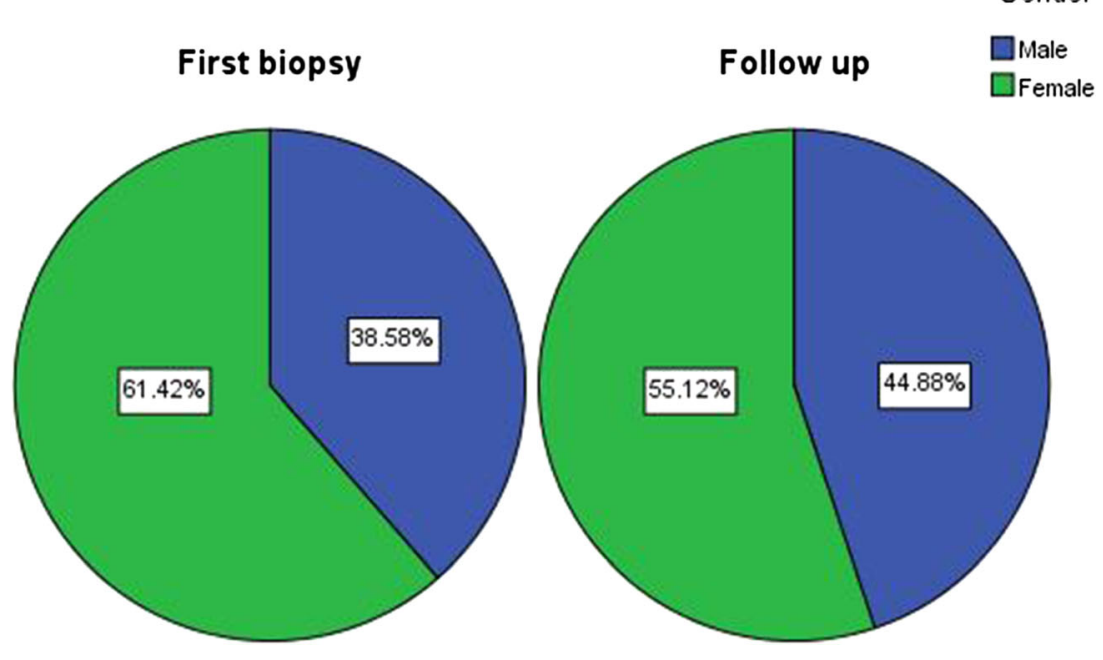

Table 3 presents the patient and biopsy parameters for the 12 cases of malignant transformation. The mean age at diagnosis of OSCC was 64.5 years, and eight of the 12 cases were in women. The average time from the initial biopsy to the diagnosis of cancer was 1.58 years. Although the floor of the mouth and border of the tongue were the predominant locations for dysplastic lesions in our study, OSCC was seen at the ridge in five cases. Four cases had no risk factors or systemic disorders.

\section{Discussion}

This study analyzed patients with mucosal lesions of OLP, OLL, dysplasia, and carcinoma in situ and their possible malignant transformation. The main criticism of studies that concentrate on the possible premalignant character of OLP and OLL is the lack of sufficient clinical and histological data to support the initial diagnosis in patients who eventually develop OSCC. In fact, clinicians and pathologists need to be experienced in diagnosing OLP. For example, Van der Meij [16] reported no consensus on histopathological diagnosis for $42 \%$ of the cases with full agreement on the clinical diagnosis of the disease. Furthermore, in $50 \%$ of the cases with such a consensus, there was a lack of clinical agreement. This problem probably occurs because OLP is likely associated with the differential diagnosis of several other diseases, such as lichenoid reactions, erythematosus lupus, leukoplakia, erythroleukoplakia, and proliferative verrucous leukoplakia [17]. Even the classic subclassification of OLP into six forms is not without problems. For example, patients can have reticular-atrophic-erosive lesions together, which makes accurate reporting difficult. Therefore, authors recently have proposed a modification of the WHO diagnostic criteria [16].

OLP and OLL are relatively common diseases that have been reported to be more frequent in females than in males

Fig. 2 Clinical appearance of the mucosal lesions at biopsy, described by different physicians

First biopsy

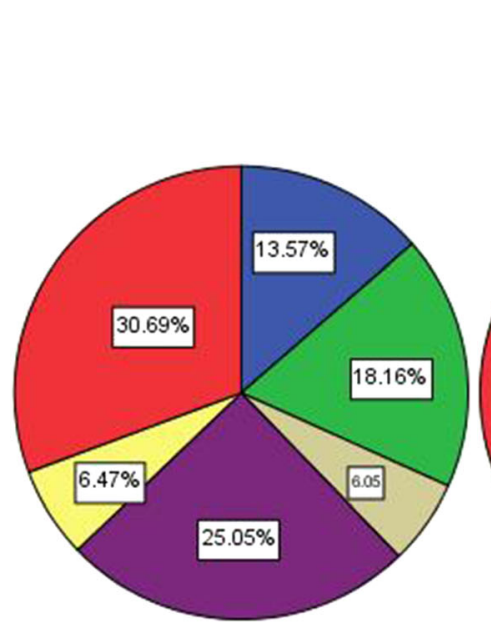

Appearance

$\square$ Reticular
$\square$ Erosive
$\square$ Ulcerative
GLichenoid
$\square$ Erythematous
$\square$ White


Fig. 3 Locations affected by mucosal lesions

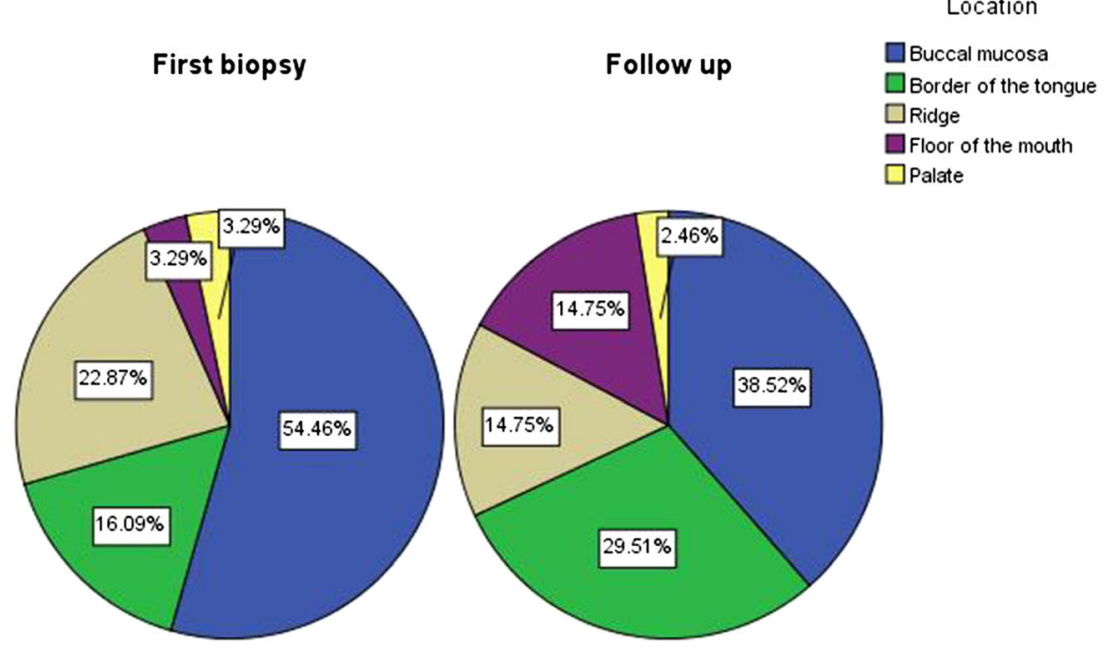

$[18,19]$. We found the same in the present study; $61.8 \%$ of the all biopsies were taken from women. Overall the more severe diagnoses were found in men, but progression towards malignancy was found in more women than men (8 of 12 cases). This is in accordance with another author's findings, who described that malignant transformation of OLP is more common in women [20]. We also agree with other authors [9] that the white lesions are the most frequent clinical forms and the buccal mucosa and tongue are the most commonly affected sites. However, regarding the severity of disease, we found that ulcerative lesions and lesions on the floor of the mouth have a significantly higher degree of dysplasia.

Only a few studies have examined the prevalence of systemic disorders in patients. As in our study, other studies found that smoking correlates significantly with the severity of diagnosis. Diabetes mellitus and hepatitis $\mathrm{C}$ are mentioned most frequently in relation to mucosal lesions [21]. Chainani-
Wu et al. [22] found that $19 \%$ of patients had arterial hypertension and $26 \%$ consumed antidepressants. Eisen et al. [23] found that $14 \%$ of patients had rheumatologic diseases. Because our study was retrospective, it was only possible to determine whether the patients had a joint complaint, and we could not obtain a more accurate diagnosis or drug treatment history. Interestingly, in our study, a positive history for joint disease corresponded significantly with the exacerbation of OLP and OLL. However, this association needs to be examined in more detail in prospective studies.

Topical steroids are the treatment of first choice for OLP. Fifty percent of our patients received topical corticosteroids and $7.8 \%$ received topical retinoids. Indeed, we found a significant reduction in the severity of dysplasia after local treatment with tretinoin in different concentrations (Retinoral ${ }^{\circledR} 0.005-0.02 \%$ ). This is in accordance with other publications that suggest that topical vitamin A may be a

Fig. 4 Diagnosis of the mucosal

Diagnosis lesions

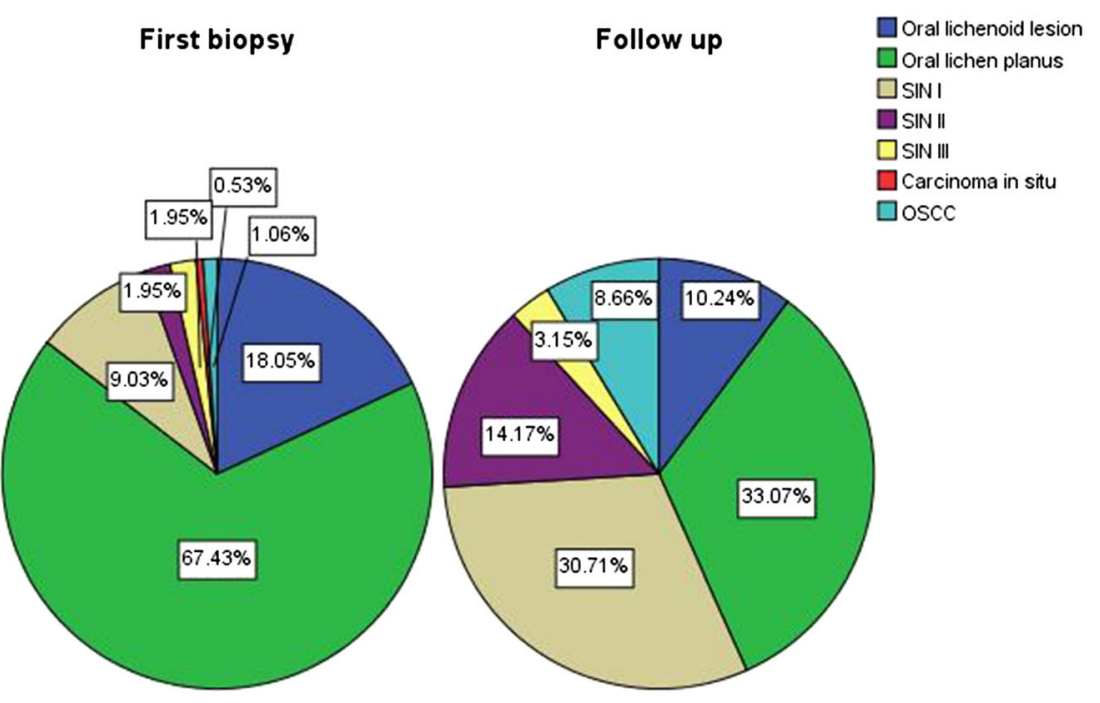


Table 2 Malignant transformation rate (MTR) in relation to the first biopsy and to all biopsies

\begin{tabular}{lllll}
\hline $\begin{array}{l}\text { Mucosal lesion } \\
\text { (first biopsy) }\end{array}$ & Cases of OSCC & $\begin{array}{l}\text { MTR in relation to } \\
\text { the first biopsy }\end{array}$ & $\begin{array}{l}\text { Mucosal lesion } \\
\text { (all biopsies) }\end{array}$ & $\begin{array}{l}\text { MTR in relation to } \\
\text { all biopsies }\end{array}$ \\
\hline OLL $(n=102)$ & 5 & $5 \%$ & OLL $(n=115)$ & $4.4 \%$ \\
OLP $(n=381)$ & 5 & $1.3 \%$ & OLP $(n=423)$ & $1.2 \%$ \\
SIN II $(n=11)$ & 1 & $9.1 \%$ & SIN II $(n=29)$ & $3.5 \%$ \\
SIN III $(n=11)$ & 1 & $9.1 \%$ & SIN III $(n=15)$ & $6.7 \%$ \\
\hline
\end{tabular}

possible treatment for mildly dysplastic OLP because of its reported usefulness in inflammatory OLP and its potential impact on mild oral dysplasia [24-26]. Further prospective studies are necessary to assess the potential value of retinoids in treating dysplasia. After retrospective analysis of published cases of supposedly malignant transformation of OLP, they concluded that many malignant transformations from OLP may be misunderstood [27]. Therefore, Zhang et al. [28] compared the genetic changes in oral dysplasia and OSCC with the results of OLPs without dysplasia. Loss of heterozygosity was detected significantly more often in dysplasia than in OLP and correlated significantly with increasing degrees of dysplasia and progression into OSCC. Within ten years, our results show an MTR of $1.2 \%$ for OLP, which represents the lowest value of all calculated MTRs. In contrast, $4.4 \%$ of the OLLs developed into OSCC, and $3.5 \%$ of SIN II and $6.7 \%$ of SIN III progressed into malignancy. These findings correspond with Zhang's statement. Other studies had similar results. For example, Van der Meij et al. [29] presented a prospective study with an MTR of $1.7 \%$; malignant transformation was found only in the OLL group. They concluded that their results supported the hypothesis that patients with OLL had an increased risk of oral cancer, but that this risk was not found for OLP.
Another problem is the lack of knowledge about how OLP transforms into OSCC. The current hypothesis is that chronic stimulation from the inflammatory and stromal cells provides the signals that cause epithelial cells to derange their growth control and, combined with oxidative stress from oxidative and nitrative products, provoke DNA damage resulting in neoplastic changes [30-33]. The advances in genetics and identification of molecular pathways and biomarkers for this pathological condition have shed new light on the complex pathogenesis of OSCC arising from OLP. But it is still unknown whether OLP develops directly into cancer or follows dysplastic progression in developing OSCC. This represents another area of research. In our study, all cases of OLP and OLL developed directly into OSCC; we found no case of a follow-up biopsy with epithelial dysplasia between the initial diagnosis of OLP or OLL and the final diagnosis of OSCC. Furthermore, it is noteworthy that during the 10-year study period, all OSCCs developed within an average of 1.58 years. Several retrospective and prospective studies have found that the MTR does not increase with a longer follow-up period [3, $11,12,15,18,23,29]$. There are short follow-up periods with high MTRs and long follow-up periods with low MTRs. Eight of our 12 patients showed risk factors such as diabetes mellitus

Table 3 Presentation of patients and biopsy parameters in 12 cases of malignant transformation: 5 from OLP, 5 from OLL, 1 from SIN II, and 1 from SIN III

\begin{tabular}{|c|c|c|c|c|c|c|c|c|}
\hline Patient & Gender & $\begin{array}{l}\text { Age }(y) \text { at } \\
\text { first diagnosis }\end{array}$ & $\begin{array}{l}\text { Systemic disorders, } \\
\text { risk factors }\end{array}$ & Appearance & Location & $\begin{array}{l}\text { First } \\
\text { diagnosis }\end{array}$ & $\begin{array}{l}\text { Age }(y) \\
\text { at diagnosis } \\
\text { of OSCC }\end{array}$ & $\Delta \mathrm{t}(\mathrm{y})$ \\
\hline 37 & $\mathrm{~F}$ & 77 & $\begin{array}{l}\text { Joint complaints, } \\
\text { mental disorder, smoker }\end{array}$ & Ulcerative & Border of the tongue & SIN II & 77 & $<1$ \\
\hline 70 & M & 43 & Diabetes mellitus, smoker & White & Palate & OLP & 44 & 1 \\
\hline 111 & M & 47 & $\begin{array}{l}\text { Diabetes mellitus, smoker, } \\
\text { alcohol consumption }\end{array}$ & White & Ridge & OLL & 47 & $<1$ \\
\hline 114 & $\mathrm{~F}$ & 65 & - & White & Buccal mucosa & OLL & 67 & 2 \\
\hline 129 & M & 50 & Smoker & Lichenoid & Buccal mucosa & OLL & 53 & 3 \\
\hline 157 & $\mathrm{~F}$ & 71 & $\begin{array}{l}\text { Joint complaints, history } \\
\text { of tumor }\end{array}$ & Ulcerative & Buccal mucosa & OLP & 74 & 3 \\
\hline 200 & $\mathrm{~F}$ & 55 & - & Ulcerative & Ridge & OLP & 57 & 2 \\
\hline 232 & $\mathrm{~F}$ & 86 & - & White & Ridge & SIN III & 86 & $<1$ \\
\hline 243 & $\mathrm{~F}$ & 83 & Joint complaints & White & Ridge & OLL & 83 & $<1$ \\
\hline 339 & $\mathrm{~F}$ & 73 & - & Lichenoid & Border of the tongue & OLL & 73 & $<1$ \\
\hline 354 & $\mathrm{~F}$ & 74 & Diabetes mellitus & Erosive & Border of the tongue & OLP & 76 & 2 \\
\hline 423 & M & 36 & Smoker & Erosive & Ridge & OLP & 37 & 1 \\
\hline
\end{tabular}


or smoking, but significant results were found only for smoking. We cannot rule out that OLP is a premalignant oral condition, but we can confirm that OLP had the lowest MTR of all diagnoses.

\section{Conclusion}

This retrospective study included 692 biopsies obtained at the Clinic of Oral Surgery, University of Zurich. The parameters of gender $(p=0.022)$ and smoking behavior $(p<0.001)$ were significantly associated with the type of histopathological diagnosis (OLL, OLP, SIN I, SIN II, SIN III, OSCC). Mucosal lesions with an ulcerative appearance $(p=0.006)$ and a location on the floor of the mouth $(p<0.001)$ showed significantly higher degrees of dysplasia or were diagnosed as OSCC. Smoking and joint disease appeared to be significant risk factors. Treatment with tretinoin in different concentrations (Retinoral ${ }^{\circledR} 0.005 \%-$ $0.02 \%)$ significantly improved diagnosis. Regarding the MTR, 12 patients (8 women, 4 men) developed OSCC within an average of 1.58 years. The OLL had a higher MTR (4.4\%) than the OLP (1.2\%). If the first biopsy showed intraepithelial neoplasia, the risk of developing OSCC greatly increased (by $3.5 \%$ for SIN II, and $6.7 \%$ for SIN III). On the basis of our results, we conclude that it is absolutely necessary to perform prospective clinical studies on the treatment of oral lesions with retinoids. Furthermore, the mutual interaction with special systemic disorders, particularly diabetes mellitus and inflammatory joint disease, appears to be of interest. However, the most important focus must be on the development of molecular or histological markers that allow a stricter differentiation to be made between the diagnoses of OLP, OLL and oral dysplasia. As a second step, we need to identify markers that allow better prognostic statements to be made concerning OSCC.

Acknowledgment The authors thank Jacquie Klesing, ELS, for the editing assistance with the manuscript.

\section{Competing interests None declared}

Ethical approval Given from the Commission of Experts, Bern, Switzerland, 9/9/2013

\section{Patient permission Not required}

Authors' contributions SC, ST, and JMB performed the retrospective study and drafted the manuscript; KJ, a dermatopathologist, participated in the design of the study; MCL and GD performed corrections of the draft; KWG and BS developed and coordinated the study design and revised the paper.

\section{References}

1. Bagan-Sebastian JV, Milian-Masanet MA, Penarrocha-Diago M, Jimenez Y (1992) A clinical study of 205 patients with oral lichen planus. J Oral Maxillofac Surg 50:116

2. Lodi G, Scully C, Carrozzo M et al (2005) Current controversies in oral lichen planus: report of an international consensus meeting. Part 1. Viral infections and etiopathogenesis. Oral Surg Oral Med Oral Pathol Oral Radiol Endod 100:40

3. Andreasen JO (1968) Oral lichen planus. 1. A clinical evaluation of 115 cases. Oral Surg Oral Med Oral Pathol 25:31

4. Eisenberg E (2000) Oral lichen planus: a benign lesion. J Oral Maxillofac Surg 58:1278

5. Jungell P, Konttinen YT, Nortamo P, Malmstrom M (1989) Immunoelectron microscopic study of distribution of T cell subsets in oral lichen planus. Scand J Dent Res 97:361

6. Khan A, Farah CS, Savage NW et al (2003) Th1 cytokines in oral lichen planus. J Oral Pathol Med 32:77

7. Farthing PM, Cruchley AT (1989) Expression of MHC class II antigens (HLA DR, DP and DQ) by keratinocytes in oral lichen planus. J Oral Pathol Med 18:305

8. Walsh LJ, Ishii T, Savage NW et al (1990) Immunohistologic analysis of epithelial cell populations in oral lichen planus. J Oral Pathol Med 19:177

9. Ismail SB, Kumar SK, Zain RB (2007) Oral lichen planus and lichenoid reactions: etiopathogenesis, diagnosis, management and malignant transformation. J Oral Sci 49:89

10. Holmstrup P, Thorn JJ, Rindum J, Pindborg JJ (1988) Malignant development of lichen planus-affected oral mucosa. J Oral Pathol 17: 219

11. Silverman S Jr, Gorsky M, Lozada-Nur F, Giannotti K (1991) A prospective study of findings and management in 214 patients with oral lichen planus. Oral Surg Oral Med Oral Pathol 72:665

12. Barnard NA, Scully C, Eveson JW et al (1993) Oral cancer development in patients with oral lichen planus. J Oral Pathol Med 22:421

13. Lo Muzio L, Mignogna MD, Favia G et al (1998) The possible association between oral lichen planus and oral squamous cell carcinoma: a clinical evaluation on 14 cases and a review of the literature. Oral Oncol 34:239

14. Mignogna MD, Lo Muzio L, Lo Russo L et al (2001) Clinical guidelines in early detection of oral squamous cell carcinoma arising in oral lichen planus: a 5-year experience. Oral Oncol 37:262

15. Bornstein MM, Kalas L, Lemp S et al (2006) Oral lichen planus and malignant transformation: a retrospective follow-up study of clinical and histopathologic data. Quintessence Int 37:261

16. van der Meij EH, van der Waal I (2003) Lack of clinicopathologic correlation in the diagnosis of oral lichen planus based on the presently available diagnostic criteria and suggestions for modifications. $J$ Oral Pathol Med 32:507

17. Sousa FA, Rosa LE (2008) Oral lichen planus: clinical and histopathological considerations. Braz J Otorhinolaryngol 74:284

18. Ingafou M, Leao JC, Porter SR, Scully C (2006) Oral lichen planus: a retrospective study of 690 British patients. Oral Dis 12:463

19. Pakfetrat A, Javadzadeh-Bolouri A, Basir-Shabestari S, Falaki F (2009) Oral lichen llanus: a retrospective study of 420 Iranian patients. Med Oral Patol Oral Cir Bucal 14:E315

20. Carbone M, Arduino PG, Carrozzo M et al (2009) Course of oral lichen planus: a retrospective study of 808 northern Italian patients. Oral Dis 15:235

21. Lodi G, Giuliani M, Majorana A et al (2004) Lichen planus and hepatitis $\mathrm{C}$ virus: a multicentre study of patients with oral lesions and a systematic review. Br J Dermatol 151:1172

22. Chainani-Wu N, Silverman S Jr, Lozada-Nur F et al (2001) Oral lichen planus: patient profile, disease progression and treatment responses. J Am Dent Assoc 132:901 
23. Eisen D (2002) The clinical features, malignant potential, and systemic associations of oral lichen planus: a study of 723 patients. J Am Acad Dermatol 46:207

24. Epstein JB, Gorsky M (1999) Topical application of vitamin A to oral leukoplakia: a clinical case series. Cancer 86:921

25. Tete S, Pappalardo S, Rubini C et al (1999) The role of apoptosis and bcl-2 protein in topical treatment of oral leukoplakia with isotretinoin. Minerva Stomatol 48:411

26. Gorsky M, Epstein JB (2002) The effect of retinoids on premalignant oral lesions: focus on topical therapy. Cancer 95:1258

27. Eisenberg E, Krutchkoff DJ (1992) Lichenoid lesions of oral mucosa. Diagnostic criteria and their importance in the alleged relationship to oral cancer. Oral Surg Oral Med Oral Pathol 73:699

28. Zhang L, Michelsen C, Cheng X et al (1997) Molecular analysis of oral lichen planus. A premalignant lesion? Am J Pathol $151: 323$
29. van der Meij EH, Schepman KP, van der Waal I (2003) The possible premalignant character of oral lichen planus and oral lichenoid lesions: a prospective study. Oral Surg Oral Med Oral Pathol Oral Radiol Endod 96:164

30. Mignogna MD, Fedele S, Lo Russo L et al (2004) Immune activation and chronic inflammation as the cause of malignancy in oral lichen planus: is there any evidence? Oral Oncol 40:120

31. Battino M, Greabu M, Totan A et al (2008) Oxidative stress markers in oral lichen planus. Biofactors 33:301

32. Kawanishi S, Hiraku Y, Pinlaor S, Ma N (2006) Oxidative and nitrative DNA damage in animals and patients with inflammatory diseases in relation to inflammation-related carcinogenesis. Biol Chem 387:365

33. Ergun S, Trosala SC, Warnakulasuriya S et al (2011) Evaluation of oxidative stress and antioxidant profile in patients with oral lichen planus. J Oral Pathol Med 40:286 